\title{
Essential oil from Zanthoxylum bungeanum Maxim. and its main components used as transdermal penetration enhancers: a comparative study*
}

\author{
Yi LAN, Hui LI, Yan-yan CHEN, Ye-wen ZHANG, Na LIU, Qing ZHANG, Qing WU ${ }^{\dagger *}$ \\ (School of Chinese Materia Medica, Beijing University of Chinese Medicine, Beijing 100102, China) \\ ${ }^{\dagger}$ E-mail: qwu@vip.sina.com \\ Received June 5, 2014; Revision accepted Aug. 7, 2014; Crosschecked Oct. 11, 2014
}

\begin{abstract}
Our previous studies had confirmed that the essential oil from Zanthoxylum bungeanum Maxim. (Z. bungeanum oil) could effectively enhance the percutaneous permeation of drug molecules as a natural transdermal penetration enhancer. The aim of the present study is to investigate and compare the skin penetration enhancement effect of $Z$. bungeanum oil and its main components on traditional Chinese medicine (TCM) active components. Toxicities of $Z$. bungeanum oil and three selected terpene compounds (terpinen-4-ol, 1,8-cineole, and limonene) in epidermal keratinocytes (HaCaT) and dermal fibroblast (CCC-ESF-1) cell lines were measured using an MTT (3-(4,5-dimethylthiazol-2-yl)-2,5-diphenyltetrazolium bromide) assay. Five model drugs in TCM external preparations, namely osthole (OT), tetramethylpyrazine (TMP), ferulic acid (FA), puerarin (PR), and geniposide (GP), which were selected based on their lipophilicity denoted by $\log K_{\mathrm{o} / \mathrm{w}}$, were tested using in vitro permeation studies in which vertical Franz diffusion cells and rat abdominal skin were employed. The secondary structure changes of skin stratum corneum (SC) and drug thermodynamic activities were investigated to understand their mechanisms of action using Fourier transform infrared (FTIR) spectroscopy and saturation solubility studies, respectively. It was found that $Z$. bungeanum oil showed lower toxicities in both HaCaT cells and CCC-ESF-1 cells compared with three terpene compounds used alone. The enhancement permeation capacities by all tested agents were in the following increasing order: terpinen-4-ol $\approx 1,8$-cineole<limonene $<Z$. bungeanum oil. The mechanisms of permeation enhancement suggested that these enhancers promoted the skin permeation of drugs mainly by affecting SC lipids. These results indicated that $Z$. bungeanum oil exhibited better performance in enhancing the skin permeation of active components in TCM preparations.
\end{abstract}

Key words: Zanthoxylum bungeanum Maxim., Essential oil, Limonene, Fourier transform infrared (FTIR) spectroscopy, Penetration enhancer, $\mathrm{HaCaT}$ doi: $10.1631 /$ jzus.B1400158

Document code: A

CLC number: R283; O65

\section{Introduction}

The pericarp of Zanthoxylum bungeanum Maxim., commonly named Huajiao in China, belongs to the Rutaceae family and has been widely used as an im-

\footnotetext{
¿ Corresponding author

* Project supported by the Beijing Natural Science Foundation (No. 7132127 ) and the Innovative Research Team in Beijing University of Chinese Medicine (No. 2011-CXTD-13), China

(C) Zhejiang University and Springer-Verlag Berlin Heidelberg 2014
}

portant spice and traditional Chinese medicine (TCM) for its special flavors and medicative characters. It is effective for the therapy of epigastric pain, dysentery, pathogenic wind, eczema, and pruritus (Wei et al., 2011). Meanwhile, the pericarp of Zanthoxylum bungeanum Maxim. is also an important herbal medicine in TCM external preparations not only because of its medicative functions described above. Based on the statement in Li Yue Pian Wen (published in 1870), a classic literature on topical remedies in China, the pericarp of $Z$. bungeanum can facilitate the 
percutaneous absorption of active components in a prescription for its unique properties, and the function mainly results from its essential oil (Huang et al., 2009). Our previous studies have also verified that the essential oil from Zanthoxylum bungeanum Maxim. (Z. bungeanum oil) can effectively promote the percutaneous permeation of two hydrophilic and lipophilic model drugs, and possesses low skin irritation potential (Lan et al., 2014). The Z. bungeanum oil is mainly composed of oxygenated monoterpenes and monoterpene hydrocarbons like terpinen-4-ol and limonene according to gas chromatography-mass spectrometry (GC-MS) analysis, and it has been reported that most of these oil contributors can enhance the transdermal permeation of the polar or non-polar drugs, such as terpinen-4-ol (Godwin and Michniak, 1999), 1,8-cineole (Narishetty and Panchagnula, 2005), limonene (Zhang et al., 2006), and linalool (Lakshmi et al., 2014). It has not been reported whether the satisfactory performance in enhancing the permeation of drugs across the skin can be achieved through using the essential oil or only one of the main oil contributors. This needs further study.

On the other hand, the active components in TCM external preparations are generally complex, and the enhancement effect of $Z$. bungeanum oil on TCM external preparations cannot be fully assessed due to the complexity of their active components, which also restricts the potential application of the natural transdermal penetration enhancer in TCM external preparations. During the past decades, the majority of quantitative structure-permeation relationship (QSPR) models were developed to provide algorithms to relate the skin permeability of compounds to their physicochemical parameters or structure descriptors, because the skin permeability of drug molecules is closely associated with their physicochemical properties, such as molecular size, lipophilicity, and solubility. Among the studies of QSPR models, Potts and Guy (1992) proposed a QSPR model that is now the most cited and applied QSPR model for predicting skin permeability: $\log k_{\mathrm{p}}=-6.3+0.71 \log K_{\mathrm{o} / \mathrm{w}}-0.0061 \mathrm{MW}$ $\left(r^{2}=0.67\right)$, where $k_{\mathrm{p}}$ is the skin permeability coefficient, $K_{\mathrm{o} / \mathrm{w}}$ is the $n$-octanol/water partition coefficient, and MW is the molecular weight. The drug lipophilicity appears to be the predominant factor affecting the skin permeability of drugs within a certain range of molecular weight (Yamashita and Hashida, 2003), and the complex active components in TCM external preparations can thus be roughly represented by model drugs with different lipophilicities. Therefore, in the present study, a series of active components with a wide range of lipophilicity, including the extremely lipophilic and hydrophilic drugs, was selected from the TCM external preparations, in an attempt to investigate the permeation enhancement activities of $Z$. bungeanum oil and the main oil contributors.

Accordingly, three main oil contributors, namely terpinen-4-ol (18.42\%), 1,8-cineole (15.49\%), and limonene $(7.47 \%)$, are selected according to GC-MS analysis previously reported by Lan et al. (2014), and the aims of the present paper are (1) to evaluate the toxicities of $Z$. bungeanum oil and three main oil contributors in skin cells, and (2) to compare their penetration enhancement activities using the TCM active components with different lipophilicities, i.e., osthole (OT) (Fu and $\mathrm{Du}, 2010)$, tetramethylpyrazine (TMP) (Han et al., 2012), ferulic acid (FA) (Li et al., 2013), puerarin (PR) (Chao, 2012), and geniposide (GP) (Sun et al., 2013). The physicochemical properties and main plant origins of these model drugs are presented in Table 1. In addition, the mechanisms of action of these enhancers were investigated.

Table 1 Plant origins and physicochemical parameters of model drugs

\begin{tabular}{llcccr}
\hline \multirow{2}{*}{ Active component } & \multicolumn{1}{c}{ Main plant origin } & $\begin{array}{l}\log K_{\mathrm{o} / \mathrm{w}} \\
\text { at } 32{ }^{\circ} \mathrm{C}\end{array}$ & $\mathrm{MW}$ & $\begin{array}{c}\text { Solubility in water } \\
\text { at } 32{ }^{\circ} \mathrm{C}(\mathrm{mg} / \mathrm{ml})\end{array}$ & $\mathrm{p} K_{\mathrm{a}}$ \\
\hline Osthole & $\begin{array}{l}\text { Cnidii Fructus (Shechuangzi); } \\
\text { Angelicae Pubescentis Radix (Duhuo) }\end{array}$ & 3.85 & 244.34 & $1.32 \times 10^{-3}$ \\
Tetramethylpyrazine & Chuanxiong Rhizoma (Chuanxiong) & 2.34 & 136.20 & 4.72 & 3.20 \\
Ferulic acid & $\begin{array}{l}\text { Angelicae Sinensis Radix (Danggui); } \\
\text { Cimicifugae Rhizoma (Shengma) }\end{array}$ & 1.26 & 194.18 & 0.54 & 4.58 \\
Puerarin & $\begin{array}{l}\text { Puerariae Lobatae Radix (Gegen) } \\
\text { Geniposide }\end{array}$ & -0.35 & 432.38 & 3.64 & 6.46 \\
& $\begin{array}{l}\text { Gardeniae Fructus (Zhizi); } \\
\text { Eucommiae Cortex (Duzhong) }\end{array}$ & -1.01 & 388.37 & 481.87 & 12.80 \\
\hline
\end{tabular}

$K_{\mathrm{o} / \mathrm{w}}: n$-octanol/water partition coefficient; MW: molecular weight; $\mathrm{p} K_{\mathrm{a}}$ : acidity strength, from the SciFinder database 


\section{Materials and methods}

\subsection{Drugs and chemicals}

Limonene, 1,8-cineole, and terpinen-4-ol, all of purity $>95.0 \%$, were obtained from TCI Chemical Industry Co., Ltd. (Shanghai, China). OT, PR, and GP were supplied by NCE Biomedical Co., Ltd. (Wuhan, China). TMP and FA were purchased from Sinopharm Chemical Reagent Co., Ltd. (Beijing, China) and Sigma-Aldrich (Shanghai, China), respectively. Propylene glycol (PG), polyoxyethylene (20) oleyl ether (Brij 98), and 3-(4,5-dimethylthiazol-2-yl)-2,5diphenyltetrazolium bromide (MTT) were obtained from Sigma-Aldrich (Shanghai, China). Acetonitrile of high performance liquid chromatography (HPLC) grade and acetic acid were supplied by Merck KGaA (Germany) and Beijing Chemical Reagent Co., Ltd. (China), respectively. All other chemicals were of the highest reagent grade available.

\subsection{Plant materials and essential oil extraction}

The dried pericarps of Zanthoxylum bungeanum Maxim. were supplied by Anguo Lulutong Co., Ltd. (Hebei, China), and identified by Prof. Peng TANG (School of Chinese Materia Medica, Beijing University of Chinese Medicine, China). A voucher specimen (No. ZB120928) was preserved in the Department of Chinese Pharmacy, Beijing University of Chinese Medicine, China. The unground $Z$. bungeanum pericarps $(2.0 \mathrm{~kg})$ were subjected to hydrodistillation in 5 volumes of deionized water for $3 \mathrm{~h}$ according to the method reported previously by Lan et al. (2014). The essential oil was collected in a yield of $2.9 \%$ $(\mathrm{w} / \mathrm{w})$ after drying under anhydrous sodium sulphate and stored at $4{ }^{\circ} \mathrm{C}$ until use.

The $Z$. bungeanum oil mainly consisted of high contents of oxygenated monoterpenes and monoterpene hydrocarbons, and the major compounds were terpinen-4-ol (18.42\%), 1,8-cineole (15.49\%), limonene (7.47\%), $\alpha$-terpineol (5.79\%), $\gamma$-terpinene $(5.62 \%)$, terpinyl acetate $(4.62 \%)$, and linalool (4.55\%) based on the GC-MS analysis (Lan et al., 2014).

\subsection{Cell line and culture}

Human skin epidermal keratinocytes (HaCaT; Fig. 1b) and dermal fibroblasts (CCC-ESF-1; Fig. 1c) were supplied by the Cell Resource Center of Institute of Basic Medical Sciences, Chinese Academy of
Medical Sciences (Peking Union Medical College, China). HaCaT cells were incubated in minimum essential medium (MEM Eagles with Earle's Balanced Salts) supplemented with $10 \%$ heat-inactivated fetal bovine serum (FBS) and $100 \mathrm{U} / \mathrm{ml}$ penicillin/ streptomycin in a humidified incubator at $37{ }^{\circ} \mathrm{C}$ and $5 \% \mathrm{CO}_{2}$. CCC-ESF-1 cells were maintained in Dulbecco's modified Eagle's medium (DME H-21 $4.5 \mathrm{~g} / \mathrm{L}$ glucose) containing $10 \% \mathrm{FBS}$ and $100 \mathrm{U} / \mathrm{ml}$ penicillin/streptomycin under the above conditions.

\subsection{HPLC methods for determination of model drugs}

The HPLC system for determining the drug content was equipped with an SPD-20A variablewavelength ultraviolet absorbance detector and two LC-20AT pumps (Shimadzu Corporation, Tokyo, Japan). A reversed phase column (Purospher STAR RP-18, $25 \mathrm{~cm} \times 4.6 \mathrm{~mm}, 5 \mu \mathrm{m}$ particle size; Merck) was employed to measure the content of TMP. The Alltima HP C18 AQ column $(25 \mathrm{~cm} \times 4.6 \mathrm{~mm}, 5 \mu \mathrm{m}$ particle size; Grace) was used to determine the contents of GP and PR. The Lichrospher RP-18e reversed phase column $(25 \mathrm{~cm} \times 4.6 \mathrm{~mm}, 5 \mu \mathrm{m}$ particle size; Merck) was selected to analyze the contents of OT and FA. The HPLC conditions were as follows: the mobile phase was a mixture of $80 / 20(\mathrm{v} / \mathrm{v})$ water/ acetonitrile and 70/30 (v/v) water (1\% acetic acid)/ acetonitrile for the assay of OT and FA, respectively, and the ultraviolet detector was set at $320 \mathrm{~nm}$ for OT and $323 \mathrm{~nm}$ for FA; The mobile phase consisted of $40 / 60(\mathrm{v} / \mathrm{v})$ water/acetonitrile for the determination of TMP, and the wavelength was set at $282 \mathrm{~nm}$; The mobile phase was a mixture of $80 / 20(\mathrm{v} / \mathrm{v})$ water/ acetonitrile for the assay of PR and GP, and the ultraviolet detector was set at $240 \mathrm{~nm}$ for PR and $251 \mathrm{~nm}$ for GP, respectively; The mobile phase was delivered at a flow rate of $1 \mathrm{ml} / \mathrm{min}$ for five drugs. The specificity, linearity, intra- and inter-day variability, and recovery of these methods were tested and satisfied the quantitative analysis request for the samples.

\subsection{Determination of drug solubility}

To determine the saturation solubility of the five model drugs in the vehicle $(80 / 20(\mathrm{v} / \mathrm{v}) \mathrm{PG} /$ water) with and without enhancers, excess drug was added to known volumes of vehicle, votexed for 5 min followed by sonication for $15 \mathrm{~min}$ to dissolve the drug, and then 
equilibrated at $(32 \pm 0.3){ }^{\circ} \mathrm{C}$ for more than $48 \mathrm{~h}$. Finally, the saturated solution was centrifuged at $10000 \mathrm{r} / \mathrm{min}$ for $10 \mathrm{~min}$. Aliquots of supernatant were filtered through a $0.22-\mu \mathrm{m}$ nylon filter and diluted with the corresponding mobile phase before HPLC analysis.

\subsection{Calculation of model drug parameters}

The five model drugs, which were common active components in TCM for external use, were selected based on their lipophilicity denoted as $\log K_{\mathrm{o} / \mathrm{w}}$. The physicochemical parameters of the drugs were obtained from literature or experimentally measured. The exact $\log K_{\mathrm{o} / \mathrm{w}}$ values of these drugs were determined with reference to a previously reported method (Chen et al., 2014). The solubilities of five drugs in water at $32{ }^{\circ} \mathrm{C}$ were measured using the method described in Section 2.5. The $\mathrm{p} K_{\mathrm{a}}$ (acidity strength) values of model drugs were obtained from the SciFinder database.

\subsection{Cytotoxicity assay}

The MTT assay was employed to determine the toxic effects of $Z$. bungeanum oil and its components on human skin epidermal keratinocytes and dermal fibroblasts in vitro. HaCaT or CCC-ESF-1 cells were seeded into 96-well plates at 7000 cells/well. After $24 \mathrm{~h}$, the cells were treated with varying concentrations of $Z$. bungeanum oil, limonene, 1,8-cineole, and terpinen-4-ol for $24 \mathrm{~h}$ at $37^{\circ} \mathrm{C}$. The cells that were incubated with a culture medium containing $1 \%$ dimethyl sulphoxide (DMSO) were used as control. Then the medium was replaced by a fresh medium containing $20 \mu \mathrm{l}$ MTT solution $(5 \mathrm{mg} / \mathrm{ml}$ in phosphate buffer) and incubated again for $4 \mathrm{~h}$. Subsequently, the medium was removed and $150 \mu \mathrm{DMSO}$ was added to dissolve the formazan crystals. The plate was incubated for $10 \mathrm{~min}$ while shaking. The microplate spectrophotometer (Thermo Scientific, Finland) was used to determine absorbance at $490 \mathrm{~nm}$. The half maximal inhibitory concentrations $\left(\mathrm{IC}_{50}\right)$ were calculated using SPSS software v16.0 with reference to the method previously reported (Xiang et al., 2010).

\subsection{Preparation of full thickness skin and stratum corneum (SC)}

Male Sprague-Dawley rats (five weeks of age, $(200 \pm 10) \mathrm{g}$ ) were supplied by SPF (Beijing) Labora- tory Animal Technology Co., Ltd., China. All the animal experiments were performed according to the approved protocols and in accordance with the Guide Principles for the Care and Use of Laboratory Animals, Beijing University of Chinese Medicine, China. The rats were anesthetized with excess ether inhalation, and the abdominal skin was excised after removal of hair by animal hair clipper (Codos, China). After removing the fat and subcutaneous tissue, the skin was cleaned with isotonic $0.01 \mathrm{~mol} / \mathrm{L}$ phosphate buffer saline (PBS; pH 7.2).

The excised skin was immersed in $4 \mathrm{~g} / \mathrm{L}$ trypsin in PBS solution at room temperature for $10 \mathrm{~h}$. The SC sheets were separated by using a cotton swab moistened with distilled water, and dried in a vacuum desiccator after cleaning with water.

\subsection{Skin permeation studies}

The vertical Franz diffusion cells (Shanghai Kaikai Technology Trade Co., Ltd., China), with a diffusional area of $1.77 \mathrm{~cm}^{2}$ and a receptor chamber volume of $7 \mathrm{ml}$, were used in skin permeation experiments. The skin prepared was sandwiched between the diffusion cells with the SC side up and the dermal side in contact with the receiver compartment. The donor chambers were filled with the drug solution $(20 \mathrm{mg} / \mathrm{ml}$ tested model drug in $80 / 20(\mathrm{v} / \mathrm{v})$ $\mathrm{PG} /$ water) and the receiver compartments with $\mathrm{pH} 7.2$ PBS for TMP, FA, PR, and GP. The 3\% Brij 98 in PBS was used as receiver solution for OT on account of its poor water solubility. The temperature and stirring rate of the diffusion apparatus were set at $32{ }^{\circ} \mathrm{C}$ and $300 \mathrm{r} / \mathrm{min}$, respectively. A $2-\mathrm{ml}$ corresponding drug solution was added into the donor compartment, and the control was treated with the vehicle only. At predetermined time intervals, an aliquot $(1 \mathrm{ml})$ of the sample was withdrawn from each receptor chamber and replaced with the equivalent volume of fresh receiver solution. All samples were determined using the corresponding HPLC method described above.

\subsection{Fourier transform infrared (FTIR) spec- troscopy studies}

The SC samples were treated with the respective enhancer solution for $12 \mathrm{~h}$ at room temperature, and the SC sheet that was incubated with the vehicle was used as control. Then SC samples were taken out, 
cleaned with distilled water to remove the residual solvent on the surface of $\mathrm{SC}$, and dried in a vacuum desiccator prior to use. The spectral measurements of all samples were made with a Nexus FTIR spectrometer (Thermo Nicolet, USA) equipped with an attenuated total reflectance (ATR) attachment under the following conditions: scanning times of 100 in the range of $650-4000 \mathrm{~cm}^{-1}$ and resolution of $2 \mathrm{~cm}^{-1}$. The spectral curves in each group were recorded using an OMNIC 6.2 program attached to the IR apparatus.

\subsection{Data analysis}

The cumulative amount of drug permeated though a unit area of skin was plotted against time (h). Steady state flux $\left(J_{\mathrm{s}}, \mu \mathrm{g} /\left(\mathrm{cm}^{2} \cdot \mathrm{h}\right)\right)$ and lag time $\left(T_{\text {lag }}, \mathrm{h}\right)$ were calculated from the slope and the $x$-intercept of the linear portion of the plot (between 6 and $12 \mathrm{~h}$ ), respectively. The cumulative amount of drugs permeating through the skin at $24 \mathrm{~h}\left(Q_{24}, \mu \mathrm{g} / \mathrm{cm}^{2}\right)$ was calculated from the drug concentration in the receiver compartments. The diffusion parameter $\left(D / h^{2}\right)$ was calculated using the following equation: $D / h^{2}=1 /\left(6 T_{\text {lag }}\right)$, where $D\left(\mathrm{~cm}^{2} / \mathrm{h}^{-1}\right)$ was the diffusion coefficient within the skin, and $h(\mathrm{~cm})$ was the diffusional path length. To compare the permeation enhancement capacities of each enhancer, the enhancement ratio (ER) was calculated as follows: $\mathrm{ER}=($ flux for skin treated with enhancer)/(flux for control).

The data were expressed as mean \pm standard deviation (SD) and the number of replicates $(n)$ was given in the pertinent figures. A two-tailed Student's $t$-test was used when comparing two different conditions. $P<0.05$ was considered to be statistically significant.

\section{Results and discussion}

\subsection{Toxicities of selected enhancers in skin cells}

The toxicities of $Z$. bungeanum oil and three main oil contributors selected were examined using MTT assay in two skin cell lines including keratinocytes and fibroblasts. The $\mathrm{IC}_{50}$ values (the concentration of drugs inducing a $50 \%$ decrease in cell viability) of all agents are summarized in Table 2, and all examined enhancers roughly induced dose-dependent reductions in cellular viability as displayed in Fig. 1a.
Table $2 \mathrm{IC}_{50}$ values of selected penetration enhancers in HaCaT keratinocyte and CCC-ESF-1 fibroblast cell lines

\begin{tabular}{lcc}
\hline \multirow{2}{*}{ Enhancer } & \multicolumn{2}{c}{$\mathrm{IC}_{50}(\mathrm{mg} / \mathrm{ml})$} \\
\cline { 2 - 3 } & $\mathrm{HaCaT}$ & $\mathrm{CCC}-\mathrm{ESF}-1$ \\
\hline Z. bungeanum oil & $2.435 \pm 0.019$ & $3.649 \pm 0.055$ \\
Limonene & $2.027 \pm 0.035^{*}$ & $0.938 \pm 0.059^{*}$ \\
Terpinen-4-ol & $0.908 \pm 0.033^{*}$ & $0.745 \pm 0.063^{*}$ \\
1,8-Cineole & $1.400 \pm 0.139^{*}$ & $1.391 \pm 0.113^{*}$ \\
\hline
\end{tabular}

Each value represents the mean $\pm \mathrm{SD}(n=6) .{ }^{*} P<0.05$, statistically significant difference between $Z$. bungeanum oil and the three selected terpene compounds

The $\mathrm{IC}_{50}$ values showed that the cellular toxicities of the examined agents were in the following decreasing order: terpinen-4-ol $>1,8$-cineole $>$ limonene $>$ $Z$. bungeanum oil (HaCaT keratinocytes) and terpinen4-ol $>$ limonene $>1,8$-cineole $>Z$. bungeanum oil (CCCESF-1 fibroblasts), respectively. It was obvious that the $\mathrm{IC}_{50}$ values of $Z$. bungeanum oil were significantly higher in both HaCaT cells and CCC-ESF-1 cells in comparison to those of all three terpene compounds, indicating that $Z$. bungeanum oil probably exhibited lower skin irritation compared with three terpene compounds used alone. However, the argument needs to be further verified by studying the irritation effects of these enhancers on animal or human skin.

Furthermore, our previous studies (Lan et al., 2014) showed that the $\mathrm{IC}_{50}$ values of Azone, a wellestablished and standard chemical penetration enhancer, were 0.047 and $0.048 \mathrm{mg} / \mathrm{ml}$ in $\mathrm{HaCaT}$ cells and CCC-ESF-1 cells, respectively, which were prominently lower than those of all three terpene compounds and suggested that the natural transdermal penetration enhancers possessed relatively low skin irritation potential due to their natural origin (Fox et al., 2011).

\subsection{Effects of penetration enhancers on percuta- neous absorption of model drugs}

To compare the penetration enhancement activities of $Z$. bungeanum oil and three selected terpene compounds through the excised rat skin, $3 \%$ enhancer solutions with model drugs were prepared by dissolving the corresponding content of the agents in a mixture of $80 / 20(\mathrm{v} / \mathrm{v})$ PG/water. PG/water was selected as a base solvent due to its negligible impact on skin tissue (Lan et al., 2014) and the ability to solubilize all tested agents, including $Z$. bungeanum oil and the highly lipophilic drug OT. Furthermore, the poor water solubility of OT was taken into consideration 

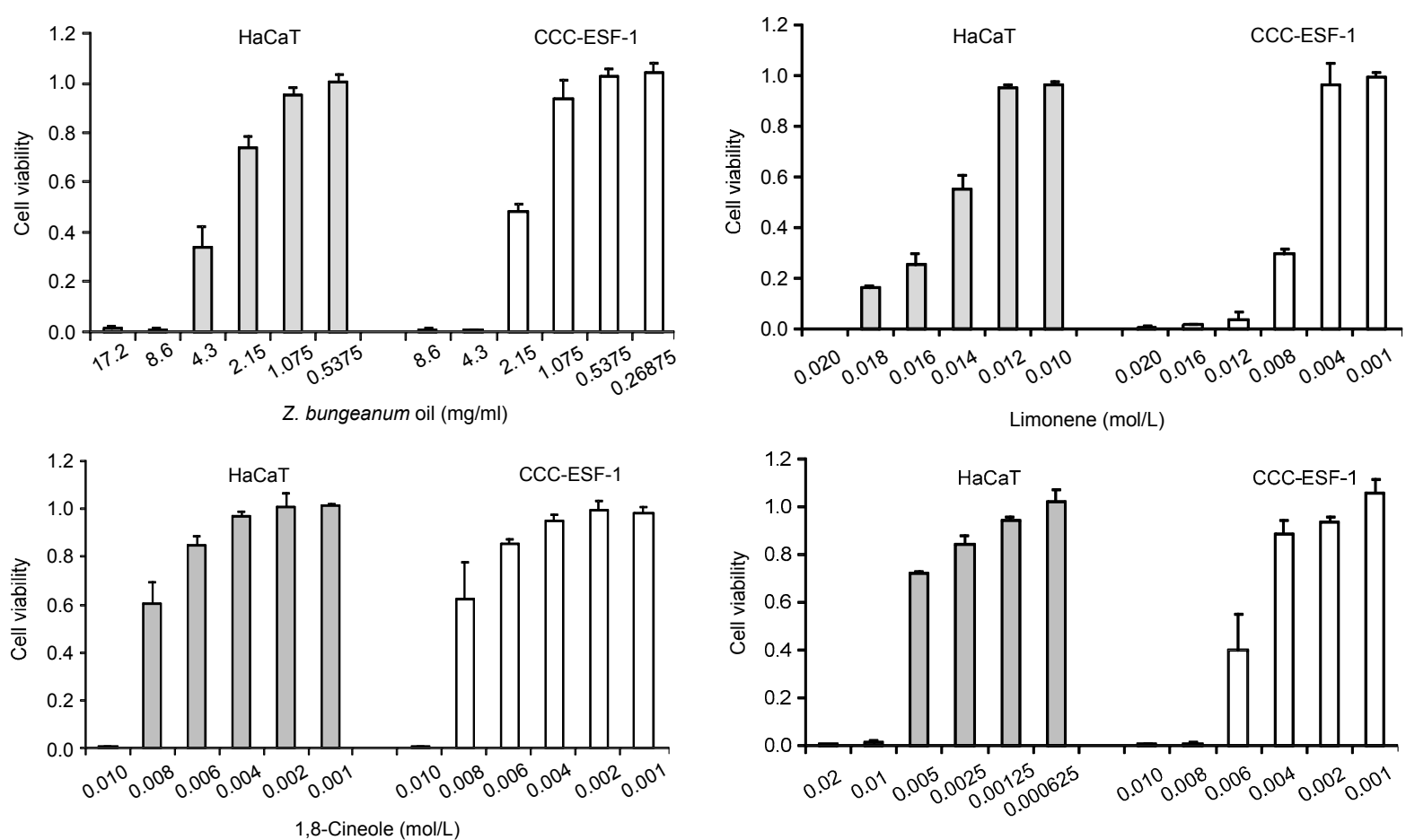

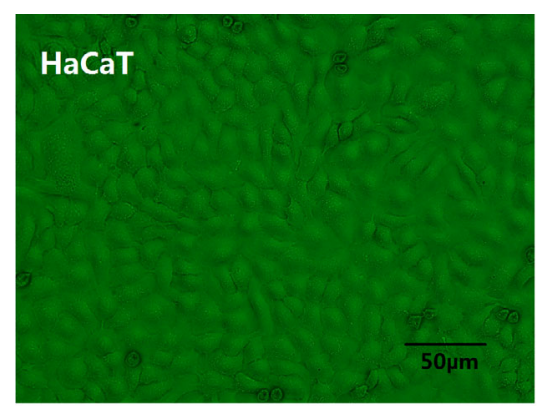

(b)

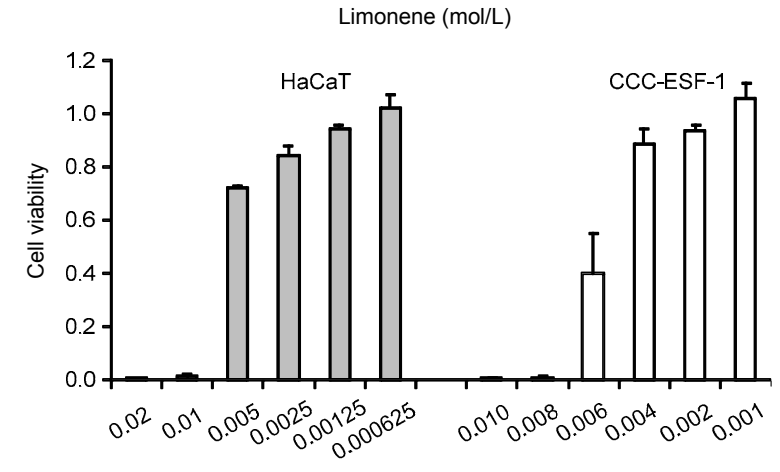

(a)

Terpinen-4-ol (mol/L)

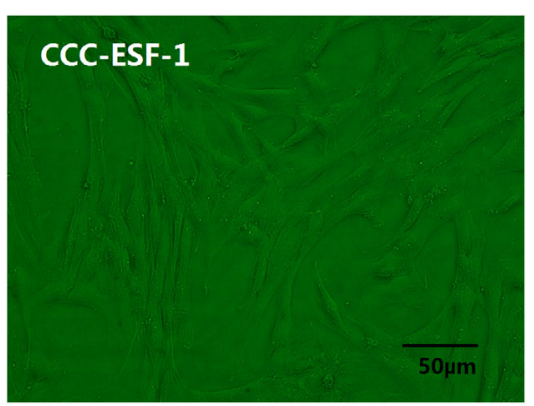

(c)

Fig. 1 Toxicity of select penetration enhancers in HaCaT keratinocyte and CCC-ESF-1 fibroblast cell lines

(a) Effect of selected enhancers on HaCaT and CCC-ESF-1 cell viabilities. Data are expressed as mean \pm SD $(n=6)$.

(b) Cellular morphology of HaCaT. (c) Cellular morphology of CCC-ESF-1

and $0.03 \mathrm{~g} / \mathrm{ml}$ Brij 98 in PBS was used as the receiver fluid to achieve the sink condition during its in vitro skin permeation process (Lan et al., 2013).

The effects of $Z$. bungeanum oil and three selected terpene compounds on the percutaneous profile and permeation parameters of model drugs (flux, $T_{\mathrm{lag}}$, $Q_{24}$, and ER values) through the excised rat skin are presented in Fig. 2 and Table 3, respectively. The $Z$. bungeanum oil significantly enhanced $(P<0.05)$ steady state fluxes or cumulative amounts of all five model drugs and produced the longer $T_{\text {lag }}$ for most drugs in comparison with the control, which showed the greatest steady state fluxes or cumulative amounts for all five model drugs among all examined agents. Meanwhile, along with the decrease of the $\log K_{\mathrm{o} / \mathrm{w}}$ values of model drugs, the permeation ER gradually increased after being treated with $Z$. bungeanum oil. This indicated that $Z$. bungeanum oil could significantly promote the percutaneous absorption of drugs with different lipophilicities, including the highly lipophilic and hydrophilic drugs. Among the three terpene compounds assayed, the highest permeation fluxes and cumulative amounts were observed with limonene for all five model drugs, while terpinen-4-ol 

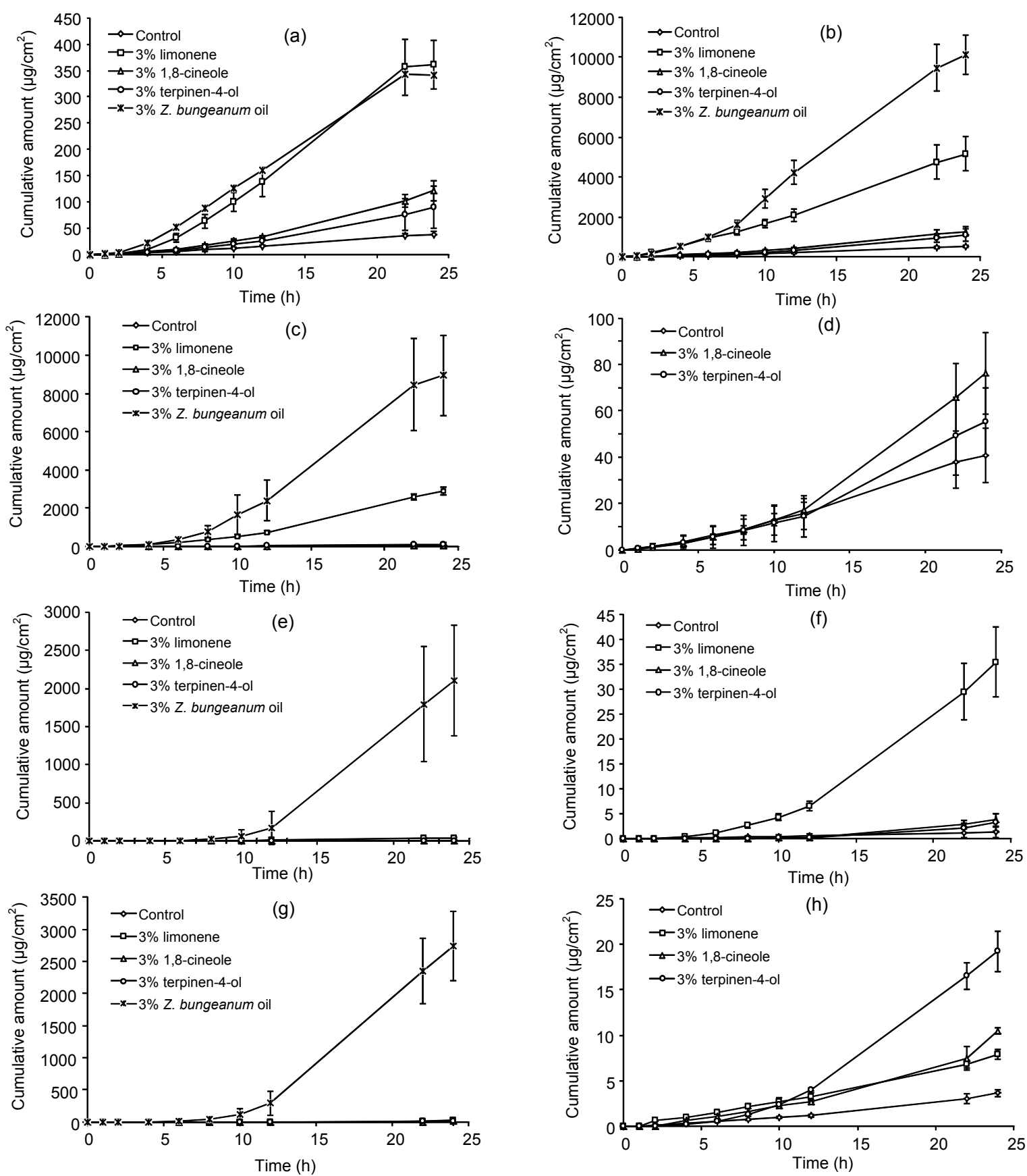

Fig. 2 Permeation profiles of five model drugs through excised rat skin

(a) Osthole (OT); (b) Tetramethylpyrazine (TMP); (c, d) Ferulic acid (FA); (e, f) Puerarin (PR); (g, h) Geniposide (GP). Data are expressed as mean $\pm \mathrm{SD}(n=5)$

and 1,8-cineole appeared to provide significant enhancement in the fluxes or cumulative amounts of more lipophilic model drugs like OT and TMP. Compared with the control, the $T_{\text {lag }}$ values of most model drugs (except TMP) were obviously increased in the presence of limonene, while there were no significant differences $(P>0.05)$ between the control and other two terpene compounds in terms of $T_{\text {lag }}$ values apart from PR. Taken as a whole, according to the permeation results of the model drugs with different lipophilicities, the enhancement permeation capacities by all examined penetration enhancers were in the following increasing order: terpinen-4-ol $\approx$ 1,8-cineole $<$ limonene $<Z$. bungeanum oil. 
Table 3 Percutaneous permeation parameters of five drug models through excised rat skin

\begin{tabular}{|c|c|c|c|c|c|c|}
\hline Drug & Enhancer & $\begin{array}{c}\text { Solubility } \\
(\mathrm{mg} / \mathrm{ml})\end{array}$ & $\begin{array}{c}\text { Flux } \\
\left(\mu \mathrm{g} /\left(\mathrm{cm}^{2} \cdot \mathrm{h}\right)\right)\end{array}$ & $\begin{array}{l}T_{\text {lag }} \\
(\mathrm{h})\end{array}$ & $\begin{array}{c}Q_{24} \\
\left(\mu \mathrm{g} / \mathrm{cm}^{2}\right)\end{array}$ & ER \\
\hline \multirow[t]{5}{*}{ OT } & Control & $9.14 \pm 0.36$ & $1.68 \pm 0.14$ & $2.79 \pm 0.61$ & $38.26 \pm 4.09$ & \\
\hline & $3 \%$ terpinen- $4-\mathrm{ol}$ & $11.18 \pm 0.24^{*}$ & $3.20 \pm 1.10^{*}$ & $3.88 \pm 1.15$ & $72.73 \pm 29.31^{*}$ & 1.90 \\
\hline & $3 \%$ 1,8-cineole & $11.96 \pm 0.35^{*}$ & $3.82 \pm 0.25^{*}$ & $3.43 \pm 0.29$ & $120.55 \pm 19.10^{*}$ & 2.27 \\
\hline & $3 \%$ limonene & $9.42 \pm 0.24$ & $17.72 \pm 3.36^{*}$ & $4.34 \pm 0.13^{*}$ & $359.91 \pm 46.33^{*}$ & 10.55 \\
\hline & $3 \%$ Z. bungeanum oil & $10.25 \pm 0.21^{*}$ & $17.90 \pm 0.94^{*}$ & $3.11 \pm 0.83$ & $339.72 \pm 22.45^{*}$ & 10.65 \\
\hline \multirow[t]{5}{*}{ TMP } & Control & $268.97 \pm 0.92$ & $20.10 \pm 2.36$ & $2.10 \pm 0.39$ & $525.36 \pm 47.46$ & \\
\hline & $3 \%$ terpinen- $4-\mathrm{ol}$ & $314.23 \pm 24.58^{*}$ & $33.00 \pm 4.50^{*}$ & $2.73 \pm 0.78$ & $1090.21 \pm 310.07^{*}$ & 1.64 \\
\hline & $3 \%$ 1,8-cineole & $319.68 \pm 23.55^{*}$ & $43.42 \pm 7.47^{*}$ & $2.26 \pm 0.29$ & $1262.84 \pm 235.47^{*}$ & 2.16 \\
\hline & $3 \%$ limonene & $308.98 \pm 25.85$ & $193.12 \pm 34.40^{*}$ & $1.24 \pm 0.34^{*}$ & $5154.02 \pm 846.68^{*}$ & 9.61 \\
\hline & $3 \%$ Z. bungeanum oil & $288.02 \pm 7.21^{*}$ & $552.08 \pm 91.75^{*}$ & $4.53 \pm 0.21^{*}$ & $10097.61 \pm 965.53^{*}$ & 27.47 \\
\hline \multirow[t]{5}{*}{ FA } & Control & $53.51 \pm 0.16$ & $1.63 \pm 0.55$ & $2.52 \pm 0.87$ & $34.21 \pm 13.99$ & \\
\hline & $3 \%$ terpinen- $4-\mathrm{ol}$ & $66.04 \pm 0.55^{*}$ & $3.29 \pm 1.43$ & $3.72 \pm 0.13$ & $123.24 \pm 110.49$ & 2.02 \\
\hline & $3 \%$ 1,8-cineole & $64.72 \pm 2.49^{*}$ & $1.99 \pm 0.33$ & $3.49 \pm 0.26$ & $76.07 \pm 17.62^{*}$ & 1.22 \\
\hline & $3 \%$ limonene & $55.65 \pm 1.17$ & $87.66 \pm 9.36^{*}$ & $3.87 \pm 0.47$ & $2874.48 \pm 214.38^{*}$ & 53.78 \\
\hline & $3 \%$ Z. bungeanum oil & $62.82 \pm 1.21^{*}$ & $352.94 \pm 178.38^{*}$ & $5.32 \pm 0.30^{*}$ & $8921.58 \pm 2102.27^{*}$ & 216.53 \\
\hline \multirow[t]{5}{*}{ PR } & Control & $126.60 \pm 0.52$ & $0.05 \pm 0.04$ & $2.59 \pm 0.61$ & $1.24 \pm 1.05$ & \\
\hline & $3 \%$ terpinen- $4-\mathrm{ol}$ & $125.38 \pm 6.98$ & $0.02 \pm 0.00$ & $4.34 \pm 1.16^{*}$ & $3.23 \pm 1.78$ & 0.40 \\
\hline & $3 \%$ 1,8-cineole & $128.63 \pm 1.72$ & $0.03 \pm 0.00$ & $5.28 \pm 0.96^{*}$ & $3.73 \pm 1.23^{*}$ & 0.60 \\
\hline & $3 \%$ limonene & $128.18 \pm 3.61$ & $0.92 \pm 0.11^{*}$ & $4.83 \pm 0.15^{*}$ & $37.88 \pm 6.06^{*}$ & 18.40 \\
\hline & 3\% Z. bungeanum oil & $129.72 \pm 0.25^{*}$ & $14.23 \pm 1.44^{*}$ & $6.65 \pm 0.16^{*}$ & $1945.07 \pm 83.29^{*}$ & 284.60 \\
\hline \multirow[t]{5}{*}{ GP } & Control & $340.78 \pm 4.59$ & $0.10 \pm 0.01$ & $0.80 \pm 0.39$ & $3.06 \pm 1.06$ & \\
\hline & $3 \%$ terpinen- $4-\mathrm{ol}$ & $345.41 \pm 1.46$ & $0.20 \pm 0.04^{*}$ & $1.47 \pm 0.83$ & $3.49 \pm 1.41$ & 2.00 \\
\hline & $3 \%$ 1,8-cineole & $345.88 \pm 2.90$ & $0.28 \pm 0.08^{*}$ & $0.49 \pm 0.05$ & $8.34 \pm 3.68$ & 2.80 \\
\hline & $3 \%$ limonene & $321.00 \pm 2.35^{*}$ & $0.57 \pm 0.02^{*}$ & $5.39 \pm 0.26^{*}$ & $19.18 \pm 2.19^{*}$ & 5.70 \\
\hline & $3 \%$ Z. bungeanum oil & $338.71 \pm 5.72$ & $46.25 \pm 31.03^{*}$ & $6.58 \pm 0.15^{*}$ & $2734.29 \pm 540.83^{*}$ & 462.50 \\
\hline
\end{tabular}

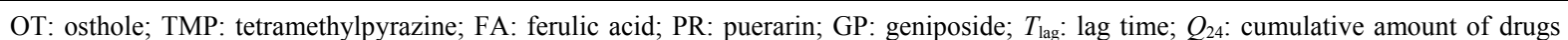
permeating through the skin at $24 \mathrm{~h}$; ER: enhancement ratio. Each value represents the mean $\pm \mathrm{SD}(n=5)$, except for ER. ${ }^{*} P<0.05$, statistically significant difference between enhancers and control for the same drug model

Further, the plots of the ERs of model drugs by penetration enhancers against the drug $\log K_{\mathrm{o} / \mathrm{w}}$ values were established as displayed in Fig. 3, and correlation analysis was performed between the ERs and lipophilicity of model drugs. The relationship for $Z$. bungeanum oil was found to be negative linear $(y=-90.24 x+310.27, r=0.946, P=0.014$; Fig. 3a), which indicated that $Z$. bungeanum oil displayed a higher capacity for the transdermal absorption of highly hydrophilic drugs. Limonene exhibited the
ERs ware in parabolic curve relationship roughly with the lipophilicity of model drugs $\left(y=-4.89 x^{2}+12.34 x+\right.$ 25.87, $r=0.682, P=0.534$; Fig. $3 b$ ), implying that limonene could achieve the optimum permeation effect for moderate lipophilic drugs (an estimated $\log K_{\mathrm{o} / \mathrm{w}}$ value of 1.0). After treatment with terpinen-4-ol or 1,8-cineole, there was no obvious relationship between their ERs and the $\log K_{\mathrm{o} / \mathrm{w}}$ values of model drugs (Figs. 3c and 3d), probably due to their relatively low penetration enhancement activities. 

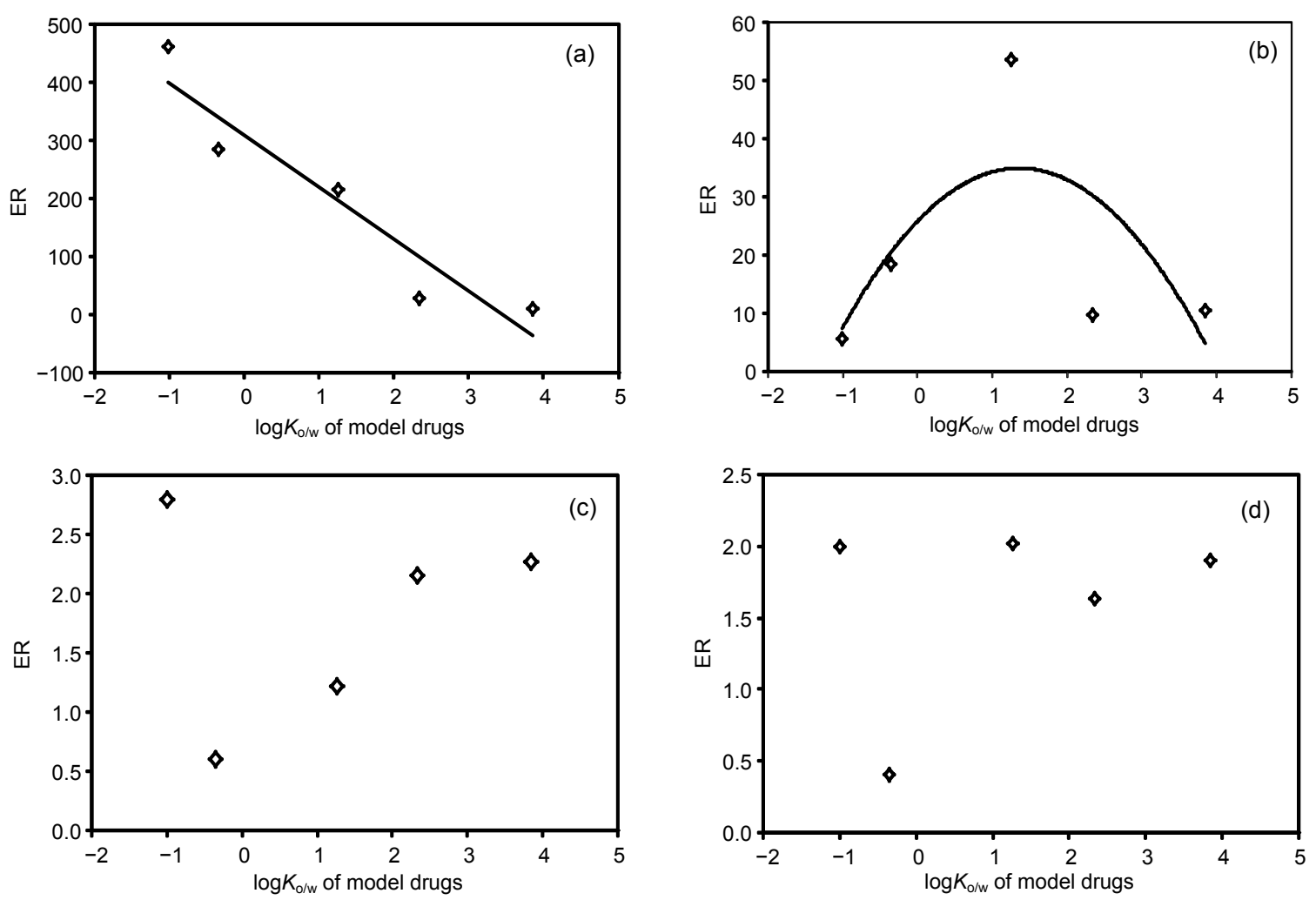

Fig. 3 Relationship between $\log K_{0 / w}$ of model drugs and enhancement ratio (ER) by penetration enhancers (a) Z. bungeanum oil; (b) Limonene; (c) 1,8-Cineole; (d) Terpinen-4-ol

The approximate transdermal permeation through human skin can be predicted from the in vitro permeation studies through excised rat skin according to their relationship studies (van Ravenzwaay and Leibold, 2004; Gu et al., 2009), though rat skin is more permeable to most compounds than human skin. Thus the rat skin can be used to gain the general insights into the enhancement permeation properties of $Z$. bungeanum oil and three selected terpene compounds.

\subsection{Possible action mechanisms}

Stratum corneum (SC), the outermost layer of skin, consists of keratin-rich corneocytes and lipids, and the lipids form bilayers surrounding the corneocytes, producing a special "brick-and-mortar" model with the corneocytes as the bricks and the intercellular lipids providing the mortar, which provides the principal rate limitation to percutaneous drug delivery (Moser et al., 2001; Williams and Barry, 2012). It is generally accepted that the barrier property mainly results from the intercellular lipids (Menon, 2002).
Data on the interaction between the enhancers and SC ingredients, especially the SC lipids, would play a crucial role in understanding their action mechanisms.

The FTIR spectrometer was employed to investigate the biophysical alterations of the skin SC on account of its advantages on obtaining information about the lipids and keratin conformation in the SC. A representative spectrum of normal rat skin SC shows the absorption peaks around 2850 and $2918 \mathrm{~cm}^{-1}$ due to symmetric and asymmetric $\mathrm{C}-\mathrm{H}$ stretching vibration of lipid alkyl chains, respectively, $-\mathrm{C}=\mathrm{O}$ peak of ester (fatty acid carbonyl stretching) at $1740 \mathrm{~cm}^{-1}$ (Jain et al., 2002), and two strong keratin amide absorption peaks in the range of $1500-1700 \mathrm{~cm}^{-1}$, namely amide I $\left(\sim 1650 \mathrm{~cm}^{-1}\right)$ and amide II $\left(\sim 1550 \mathrm{~cm}^{-1}\right)$ (Moore and Rerek, 2000). These peak positions and peak areas are closely associated with the structure and amounts of corresponding SC ingredients, respectively. The alterations in peak position of lipid alkyl chains or amides are always accompanied by the structure changes of corresponding ingredients, and 
the reduction of the SC lipids or keratins can cause the decrease in peak areas of lipids or amides. In addition, the frequencies of the fatty acid carbonyl stretching mode, amide I and amide II modes are sensitive to hydrogen bonding in the polar head groups of SC lipids, and the hydrogen bonding plays an important role in the bilayer structure and integrity of lipids.

The FTIR absorption spectra of rat skin SC treated with $Z$. bungeanum oil and three selected terpene compounds are displayed in Fig. 4. The obvious shift in peak position or change in peak area was not observed after being treated with the solvent, indicating that $80 / 20(\mathrm{v} / \mathrm{v}) \mathrm{PG} /$ water had negligible effect on the SC lipids or keratins (as shown in Table 4 and Fig. 5). All examined penetration enhancers resulted in no obvious change in symmetric $\mathrm{C}-\mathrm{H}$ stretching apart from 1,8-cineole or Z. bungeanum oil, while they caused the shift of asymmetric $\mathrm{C}-\mathrm{H}$ stretching to higher wavenumber. Limonene and $Z$. bungeanum oil induced the relatively wide shift of approximately 5.5 and $2.5 \mathrm{~cm}^{-1}$, respectively, and the decreasing trend of shift in peak position was limonene $>Z$. bungeanum oil $>$ terpinen-4-ol $\approx 1,8$-cineole. Meanwhile, these enhancers induced significant reductions in the peak areas of asymmetric and symmetric $\mathrm{C}-\mathrm{H}$ stretching except 1,8-cineole, and the decreasing trend in peak area reduction was $Z$. bungeanum oil $>$ limonene $>$ terpinen-4-ol $>1,8$-cineole. These results were roughly in accordance with the enhancement permeation capacities by all examined agents, indicating that these enhancers promoted the drug permeation mainly by perturbing and extracting the SC lipids. In addition, the presence of $Z$. bungeanum oil and limonene resulted in the absence of $\mathrm{a}-\mathrm{C}=\mathrm{O}$ peak of fatty acid, which implied that they could interact with the head polar regions of the SC lipids, resulting in the increased skin permeability, to some extent, due to the disorder of integrity of SC lipids. The shift of amide II to high wavenumber was observed after treatment with 1,8-cineole, while

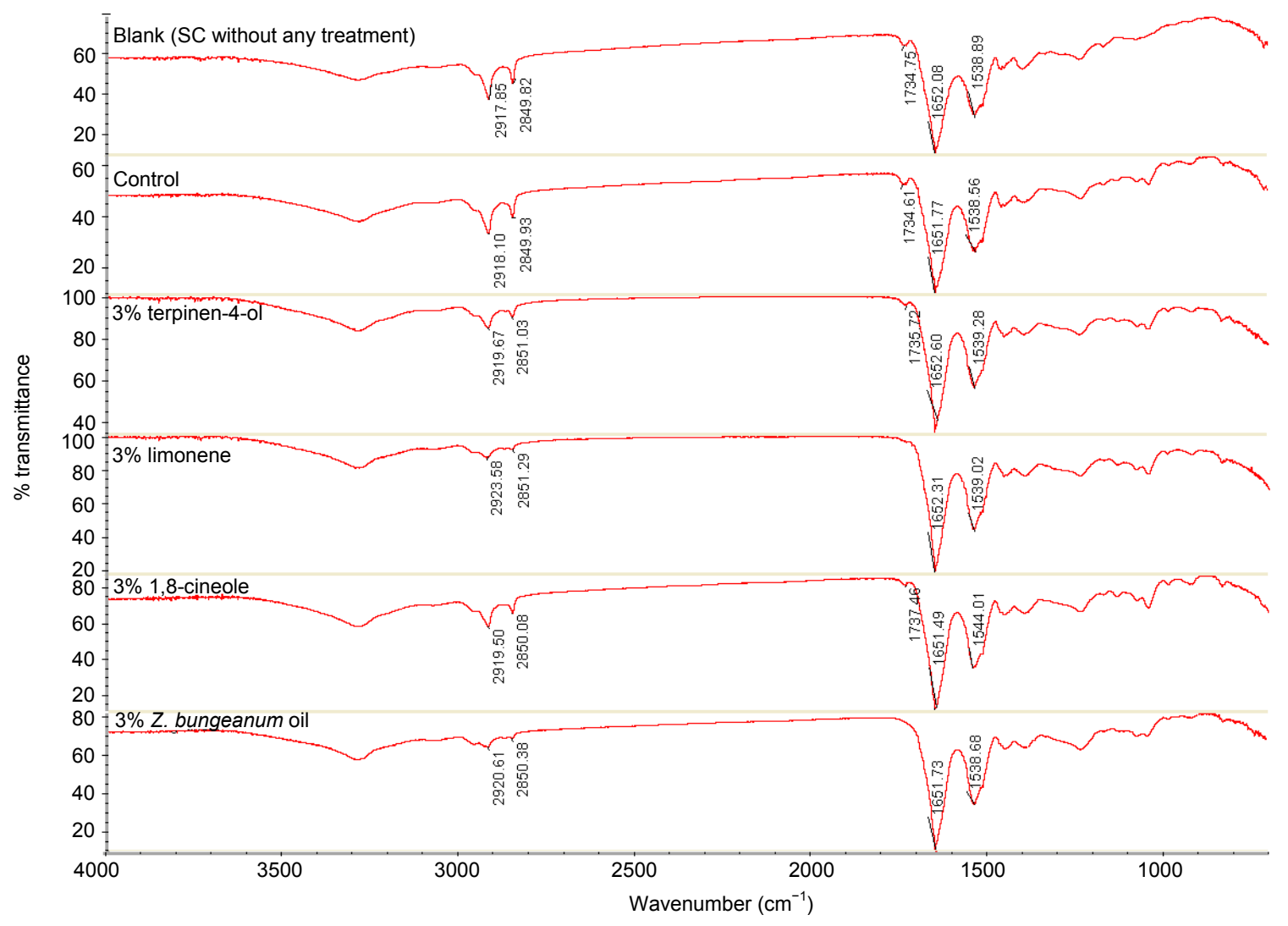

Fig. 4 FTIR absorption spectra of rat SC treated with various agents for $12 \mathrm{~h}$ 
Table 4 Peak positions of $\mathrm{C}-\mathrm{H}$ stretching of lipids and amides of keratin treated with various agents

\begin{tabular}{lcccc}
\hline \multirow{2}{*}{ Enhancer } & \multicolumn{4}{c}{ Peak position $\left(\mathrm{cm}^{-1}\right)$} \\
\cline { 2 - 5 } & $\begin{array}{c}\text { Asymmetric C-H } \\
\text { stretching of lipids }\end{array}$ & $\begin{array}{c}\text { Symmetric C-H } \\
\text { stretching of lipids }\end{array}$ & Amide I & Amide II \\
\hline Blank & 2917.85 & 2849.82 & 1652.08 & 1538.89 \\
Control & 2918.10 & 2849.93 & 1651.77 & 1538.56 \\
3\% limonene & 2923.58 & 2851.29 & 1652.31 & 1539.02 \\
3\% 1,8-cineole & 2919.50 & 2850.08 & 1651.49 & 1544.01 \\
3\% terpinen-4-ol & 2919.67 & 2851.03 & 1652.60 & 1539.28 \\
3\% Z. bungeanum oil & 2920.61 & 2850.38 & 1651.73 & 1538.68 \\
\hline
\end{tabular}

Blank was SC without any treatment

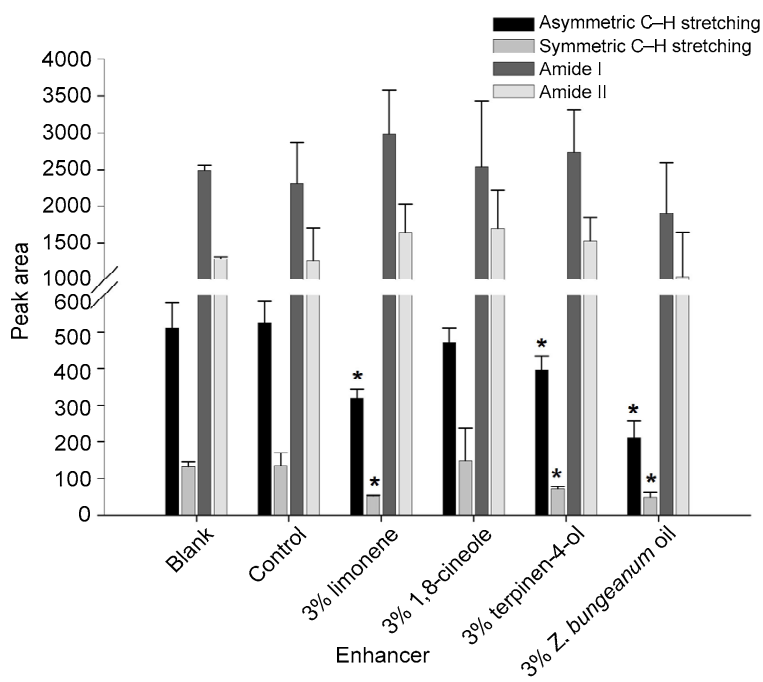

Fig. 5 Peak areas of $\mathrm{C}-\mathrm{H}$ stretching of SC lipids and amides of keratin treated with various agents for $12 \mathrm{~h}$ Blank was SC without any treatment. Data are expressed as mean \pm SD $(n=5) .{ }^{*} P<0.05$, statistically significant difference between enhancers and control

it had no significant effect on the peak area of amide II, suggesting that it could alter the keratin conformation to some extent. The other three enhancers appeared to have no obvious effect on keratin according to the negligible change in peak position or peak area of lipids and amides.

Furthermore, the drug thermodynamic activities probably were altered in the presence of these enhancers on account of their lipophilic properties. The saturation solubilities of model drugs with and without $Z$. bungeanum oil or three terpene compounds were thus measured to investigate the effect of these enhancers on thermodynamic activities of model drugs (Narishetty and Panchagnula, 2004). As evident from Table $3, Z$. bungeanum oil could significantly increase the saturation solubilities of OT, TMP, FA, and PR $(P<0.05)$, while it did not obviously alter the saturation solubility of GP in comparison to the control $(P>0.05)$. Similarly, both terpinen-4-ol and 1,8 -cineole could obviously increase the saturation solubilities of the more lipophilic drugs (OT, TMP, and FA; $P<0.05$ ), and had negligible effect on the saturation solubilities of the hydrophilic drugs (PR and GP; $P>0.05$ ), which indicated that the thermodynamic activities of the more lipophilic drugs were changed in the presence of terpinen-4-ol, 1,8-cineole, and $Z$. bungeanum oil. However, limonene had no obvious effect on these lipophilic drugs like OT and TMP $(P>0.05)$, and significantly decreased the saturation solubility of highly hydrophilic drug GP $(P<0.05)$. Results of this study suggested that these enhancers promoted the percutaneous absorption of drugs, especially the more lipophilic drugs, partially by altering drug thermodynamic activities due to their lipophilic properties.

\section{Conclusions}

The $Z$. bungeanum oil exhibited lower toxicities in both $\mathrm{HaCaT}$ keratinocytes and CCC-ESF-1 fibroblasts compared with three studied terpene compounds or chemical penetration enhancer Azone, which indicated that the natural TCM penetration enhancer would be helpful to decrease skin irritation. The skin permeation studies revealed the enhancement permeation capacities by all examined penetration enhancers in the following increasing order: 
terpinen-4-ol $\approx 1,8$-cineole $<$ limonene $<Z$. bungeanum oil. Meanwhile, the $Z$. bungeanum oil displayed a higher capacity for the transdermal permeation of highly hydrophilic drugs, and limonene could achieve the optimum permeation effect for moderate lipophilic drugs. The results of FTIR and saturation solubility studies indicated that these enhancers promoted the percutaneous absorption of drugs mainly by affecting the SC lipids, and the increased percutaneous permeation of lipophilic drugs partially resulted from the alterations of drug thermodynamic activities due to the lipophilic properties of these penetration enhancers.

\section{Acknowledgements}

The authors thank Mr. Zi-jian WANG (Center of Science Experiment, Beijing University of Chinese Medicine, Beijing, China) for his assistance in the FTIR analysis.

\section{Compliance with ethics guidelines}

Yi LAN, Hui LI, Yan-yan CHEN, Ye-wen ZHANG, Na LIU, Qing ZHANG, and Qing WU declare that they have no conflict of interest.

All institutional and national guidelines for the care and use of laboratory animals were followed.

\section{References}

Chao, Z., 2012. Effect of Azone on in vitro transdermal absorption of puerarin from Guping cataplasmata. Chin. J. Exp. Tradit. Med. Form., 18(6):1-3 (in Chinese).

Chen, X., Zhang, Z., Liu, D., et al., 2014. Study on preparation of salvianolic acid phospholipid compound. Chin. J. Chin. Mater. Med., 39(2):216-221 (in Chinese). [doi:10.4268/ cjcmm20140211]

Fox, L.T., Gerber, M., Plessis, J.D., et al., 2011. Transdermal drug delivery enhancement by compounds of natural origin. Molecules, 16(12):10507-10540. [doi:10.3390/ molecules 161210507]

Fu, Y.H., Du, H.B., 2010. The experimental study on transdermal absorption in vitro of osthol in Huoxie Zhitong patch. Med. Inform., 23(4):896-897.

Godwin, D.A., Michniak, B.B., 1999. Influence of drug lipophilicity on terpenes as transdermal penetration enhancers. Drug Dev. Ind. Pharm., 25(8):905-915. [doi:10. 1081/DDC-100102251]

Gu, S., Gao, J., Hou, X., et al., 2009. Effects of penetration enhancers on Shuangwu traumatic formula: in vitro percutaneous absorption and in vivo pharmacodynamic evaluation of an herb medicine. Eur. J. Pharm. Biopharm., 73(3):385-390. [doi:10.1016/j.ejpb.2009.08.001]

Han, J.Z., Sun, J., Zhu, Q.G., et al., 2012. A modified LC-MS/MS method for determination of tetramethylpyrazine in microdialysis samples and calibration of home-made linear probes. Biomed. Chromatogr., 26(10):1276-1281. [doi:10.1002/bmc.2689]

Huang, L., Gu, Y., Li, H., 2009. Advances in herbal volatile oil and aromatic herbs. Chin. J. Chin. Mater. Med., 34(12): 1605-1611 (in Chinese).

Jain, A.K., Thomas, N.S., Panchagnula, R., 2002. Transdermal drug delivery of imipramine hydrochloride: I. Effect of terpenes. J. Control. Release, 79(1-3):93-101. [doi:10. 1016/S0168-3659(01)00524-7]

Lakshmi, P., Mounika, K., Saroja, C., 2014. Transdermal permeation enhancement of lamotrigine using terpenes. Pharma Care Health Sys., 1:103. [doi:10.4172/jpchs. 1000103]

Lan, Y., Wang, Q., An, J., et al., 2013. Effect of different receiving solution on in vitro drug transdermal test. Chin. J. Exp. Tradit. Med. Form., 19(13):7-10 (in Chinese).

Lan, Y., Wu, Q., Mao, Y.Q., et al., 2014. Cytotoxicity and enhancement activity of essential oil from Zanthoxylum bungeanum Maxim. as a natural transdermal penetration enhancer. J. Zhejiang Univ.-Sci. B (Biomed. \& Biotechnol.), 15(2):153-164. [doi:10.1631/jzus.B1300230]

Li, X., Wang, Q., Lan, Y., et al., 2013. Effect of microemulsion technology on release of compounds in Zhitong cataplasm and content variation. China J. Chin. Mater. Med., 38(16):2614-2617 (in Chinese). [doi:10. 4268/cjcmm20131615]

Menon, G.K., 2002. New insights into skin structure: scratching the surface. Adv. Drug Deliv. Rev., 54:S3-S17. [doi:10.1016/S0169-409X(02)00121-7]

Moore, D.J., Rerek, M.E., 2000. Insights into the molecular organization of lipids in the skin barrier from infrared spectroscopy studies of stratum corneum lipid models. Acta Derm. Venereol., 208(Suppl.):16-22. [doi:10.1080/ 000155500750042817]

Moser, K., Kriwet, K., Naik, A., et al., 2001. Passive skin penetration enhancement and its quantification in vitro. Eur. J. Pharm. Biopharm., 52(2):103-112. [doi:10.1016/ S0939-6411(01)00166-7]

Narishetty, S.T.K., Panchagnula, R., 2004. Transdermal delivery of zidovudine: effect of terpenes and their mechanism of action. J. Control. Release, 95(3):367-379. [doi:10.1016/j.jconrel.2003.11.022]

Narishetty, S.T.K., Panchagnula, R., 2005. Effect of L-menthol and 1,8-cineole on phase behavior and molecular organization of SC lipids and skin permeation of zidovudine. J. Control. Release, 102(1):59-70. [doi:10. 1016/j.jconrel.2004.09.016]

Potts, R.O., Guy, R.H., 1992. Predicting skin permeability. Pharm. Res., 9(5):663-669. [doi:10.1023/A:10158103 12465]

Sun, M.J., You, W.L., Zhang, J., et al., 2013. Preparation and stability of geniposide cream. Chin. Pharm. J., 48(6): 455-460.

van Ravenzwaay, B., Leibold, E., 2004. A comparison between in vitro rat and human and in vivo rat skin absorption studies. Human Exp. Toxicol., 23(9):421-430. [doi:10.1191/0960327104ht471oa] 
Wei, S.G., Zhang, H.H., Wang, Y.Q., et al., 2011. Ultrasonic nebulization extraction-heating gas flow transferheadspace single drop microextraction of essential oil from pericarp of Zanthoxylum bungeanum Maxim. $J$. Chromatogr. A, 1218(29):4599-4605. [doi:10.1016/j. chroma.2011.05.047]

Williams, A.C., Barry, B.W., 2012. Penetration enhancers. Adv. Drug Deliv. Rev., 64(Suppl.):128-137. [doi:10. 1016/j.addr.2012.09.032]

Xiang, W., Gao, A., Liang, H., et al., 2010. Reversal of p-glycoprotein-mediated multidrug resistance in vitro by milbemycin compounds in adriamycin-resistant human breast carcinoma (MCF-7/adr) cells. Toxicol. in Vitro, 24(6):1474-1481. [doi:10.1016/j.tiv.2010.07.020]

Yamashita, F., Hashida, M., 2003. Mechanistic and empirical modeling of skin permeation of drugs. Adv. Drug Deliv. Rev., 55(9):1185-1199. [doi:10.1016/S0169-409X(03)00 118-2]

Zhang, C.F., Yang, Z.L., Luo, J.B., 2006. Effects of enantiomer and isomer permeation enhancers on transdermal delivery of ligustrazine hydrochloride. Pharm. Dev. Technol., 11(4):417-424. [doi:10.1080/10837450600770163]

\section{中文概要:}

\section{本文题目: 花椒挥发油与其主要成分经皮促透作用的比较研究}

Essential oil from Zanthoxylum bungeanum Maxim. and its main components used as transdermal penetration enhancers: a comparative study

研究目的: 比较花椒挥发油与其主要成分作为经皮促透剂的皮肤细胞毒性, 对系列不同油水分配系数药 物促透作用特征及其作用机制。

创新要点: 选择系列不同油水分配系数中药活性成分简化并表征中药制剂中复杂有效成分, 对比研究花 椒挥发油与其主要成分的经皮促透作用特征差异。

研究方法: 利用表皮角质形成细胞 (HaCaT) 和真皮成纤维细胞 (CCC-ESF-1) 测定其皮肤细胞毒性, 选 择系列不同油水分配系数中药活性成分作为模型药物测定花椒挥发油与其主要成分的经皮促 透作用特征差异，并在此基础上采用傅利叶变换红外光谱（FTIR）等研究其促透作用机制。

重要结论: 花椒挥发油相对于其单一组成成分具有更好的经皮促透效果。

关键词组：花椒挥发油；柠檬烯；促透剂；表皮角质形成细胞（HaCaT）; 傅利叶变换红外光谱（FTIR） 